INPLASY

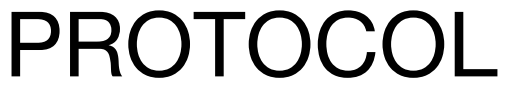

To cite: Yu et al. Diagnostic accuracy of Mycobacterium tuberculosis cell free DNA for tuberculosis: A protocol of systematic review and metaanalysis. Inplasy protocol 2020110101. doi: 10.37766/inplasy2020.11.0101

Received: 23 November 2020

Published: 23 November 2020

Corresponding author:

Yan Shi

hiyanjhk@163.com

Author Affiliation: Zhejiang Chinese Medicine and Western Medicine Integrated Hospital

Support: None.

Review Stage at time of this submission: Data extraction.

Conflicts of interest: None.

\section{Diagnostic accuracy of Mycobacterium tuberculosis cell free DNA for tuberculosis: A protocol of systematic review and meta-analysis}

Yu, G1; Shen, Y2; Ye, B3; Shi, Y4.

Review question / Objective: Early clinical identification tuberculosis (TB) infections continue to be a major challenge. Mycobacterium tuberculosis cell free DNA (cfDNA) have the ability to rapidly detect TB The purpose of this study was to evaluate the comprehensive evidence for the use of cfDNA for rapid TB diagnosis.

Condition being studied: Early diagnosis of TB is still difficult, which is detrimental to TB control. cfDNA is an innovative TB test with excellent diagnostic efficacy in the early diagnosis of TB, especially in low bacterial load TB.

Information sources: We will search candidate studies that assessing the accuracy of cfDNA for diagnosis of TB through PubMed, Embase and the Cochrane Library. References cited in a review or meta-analysis will also be evaluated to identify additional studies.

INPLASY registration number: This protocol was registered with the International Platform of Registered Systematic Review and Meta-Analysis Protocols (INPLASY) on 23 November 2020 and was last updated on 23 November 2020 (registration number INPLASY2020110101).

\section{INTRODUCTION}

Review question / Objective: Early clinical identification tuberculosis (TB) infections continue to be a major challenge. Mycobacterium tuberculosis cell free DNA (cfDNA) have the ability to rapidly detect TB The purpose of this study was to evaluate the comprehensive evidence for the use of cfDNA for rapid TB diagnosis.

Rationale: cfDNA have the ability to rapidly detect TB.

Condition being studied: Early diagnosis of TB is still difficult, which is detrimental to 
TB control. cfDNA is an innovative TB test with excellent diagnostic efficacy in the early diagnosis of TB, especially in low bacterial load TB.

\section{METHODS}

Search strategy: \#1 "Tuberculosis"[Mesh] OR "Tuberculoses Kochs Disease" OR "Koch's Disease" OR "Koch Disease" OR "Mycobacterium tuberculosis Infection" OR "Infection, Mycobacterium tuberculosis" OR "Infections, Mycobacterium tuberculosis" OR "Mycobacterium tuberculosis Infections" \#2 "Cell-Free Nucleic Acids"[Mesh] OR "Cell Free Nucleic Acids" OR "Nucleic Acids, CellFree" OR "Circulating Cell-Free Nucleic Acids" OR "Circulating Cell Free Nucleic Acids" OR "Circulating Nucleic Acids" OR "Acids, Circulating Nucleic" OR "Nucleic Acids, Circulating" OR "Cell-Free Nucleic Acid" OR "Cell Free Nucleic Acid" OR "Nucleic Acid, Cell-Free" OR "Cell-Free DNA" OR "Cell Free DNA" OR "DNA, CellFree" OR cfDNA OR cirDNA OR "Cell-Free Deoxyribonucleic Acid" OR "Acid, Cell-Free Deoxyribonucleic" OR "Cell Free Deoxyribonucleic Acid" OR "Deoxyribonucleic Acid, Cell-Free" OR "Circulating DNA" OR "DNA, Circulating" OR "Cell-Free RNA" OR "Cell Free RNA" OR "RNA, Cell-Free" OR cfRNA OR cirRNA OR "Cell-Free Ribonucleic Acid" OR "Acid, Cell-Free Ribonucleic" OR "Cell Free Ribonucleic Acid" OR "Ribonucleic Acid, Cell-Free" OR "Circulating RNA" OR "RNA, Circulating" \#3 \#1 AND \#2.

Participant or population: Participants with suspected TB infection, who are confirmed the diagnosis by cfDNA. Participants of any ethnicity, sex, or age will be included.

Intervention: cfDNA will be identified as index tests.

Comparator: Single-arm studies will also be included if participants, index tests, outcomes meet the inclusion criteria. Comparator tests are not a mandatory indicator.
Study designs to be included: Any type of study design such as retrospective studies, prospective studies, case-control studies, if the study had assessed the efficacy of cfDNA in diagnosing TB. Studies that report only sensitivity or specificity will be excluded.

Eligibility criteria: Original studies that diagnosed TB by cfDNA and have access to the full text will be included in this systematic review and meta-analysis. Clear reference standards in the original study. True positive (TP), false positive (FP), false negative (FN), and true negative (TN) values for the index tests could be extracted directly or calculated from the original studies. Studies reported in languages other than English, Studies with less than 10 specimens, conference abstracts without full articles, and case reports will be excluded.

Information sources: We will search candidate studies that assessing the accuracy of cfDNA for diagnosis of TB through PubMed, Embase and the Cochrane Library. References cited in a review or meta-analysis will also be evaluated to identify additional studies.

Main outcome(s): The sensitivity and specificity of the index tests will be considered as the main outcome. Sensitivity is the probability that the index tests will detect positive in an infected patient. Specificity is the probability that the index tests will detect negative in a noninfected patient.

Data management: We will use ENDNOTE X9.2 literature management software to manage related literature. The search candidate studies will be imported into the software. Two independent researchers (GCY and YQS) will evaluate the candidate studies for inclusion based on inclusion criteria. They will review the title and abstract first, then the full text. If there is disagreement between the two researchers, they will consult with a third investigator (LHL). We will extract information for each study such as first author; publication date; country; type of 
study design; patient selection method; TP, $F P, F N$, and TN values for the index tests; different types of study design; patient selection method; specimen types; target gene; test method; specimen condition; TB types along with other parameters. As with the literature screening process, the same two researchers will perform the data extraction independently, and if there is any disagreement, it will be handled in the same way as in the literature screening phase.

Quality assessment / Risk of bias analysis: Following a revised tool for Quality Assessment of Diagnostic Accuracy Studies (QUADAS-2), two researchers will independently evaluate the study quality. The discrepancy will be handled in the same way as in the data extraction phase. Publication bias assessment is not required by the PRISMA-DTA statement. The Grading of Recommendations Assessment, Development and Evaluation (GRADE) guideline will be used to assess the strength of the body of evidence.

Strategy of data synthesis: TP, FP, FN, and TN values for the index tests in each included study will be obtained first, then the estimated pooled sensitivity and specificity of NAAT associated with the $95 \%$ confidence interval (CI) will be calculated using MedCalc Statistical Software version 15.2.2 (MedCalc Software bvba, Ostend, Belgium; http:// www.medcalc.org; 2015). Forest plots for sensitivity and specificity will be generated for each study. The heterogeneity will be determined by using the 12 statistic. $12=$ $0 \%$ will indicate no observed heterogeneity, $12<50 \%$ will be considered minor heterogeneity, $12>50 \%$ will be considered substantial heterogeneity

Subgroup analysis: When significant heterogeneity is observed, subgroup, metaregression and sensitivity analyses will be used to explore sources of heterogeneity. Subgroup and meta-regression analyses will perform on different types of study design; patient selection method; specimen types; target gene; test method; specimen condition; TB types.
Sensibility analysis: When significant heterogeneity is observed, sensitivity analyses will be used to explore sources of heterogeneity.

\section{Country(ies) involved: China.}

Keywords: Tuberculosis, diagnostic accuracy, cfDNA, Polymerase Chain Reaction, meta-analysis.

Contributions of each author:

Author 1 - Guocan Yu - The author searched databases, selected literatures, managed data, assessed quality, drafted and revised the manuscript.

Email: dabaitwo@163.com

Author 2 - Yanqin Shen - The author searched databases, selected literatures, managed data and evaluated quality.

Email: yanqinshen@yeah.net

Author 3 - Bo Ye - The author drafted and revised the manuscript.

Email: yeboboye@126.com

Author 4 - Yan Shi - The author provided statistical expertise, read, feedback and approved the final manuscript.

Email: shiyanjhk@163.com 